\title{
Combining dynamic and static robotic telepathology: a report on 184 consecutive cases of frozen sections, histology and cytology
}

\author{
Vincenzo Della Mea*, Palmina Cataldi, \\ Barbara Pertoldi and Carlo A. Beltrami \\ Institute of Pathology, University of Udine, Italy
}

Received 1 July 1999

Accepted 4 February 2000

The aim of this paper is to describe the experiments carried out to evaluate the diagnostic efficacy of a dynamic-robotic telepathology system for the delivery of pathology services to distant hospitals. The system provides static/dynamic features and the remote control of a robotized microscope over 4 ISDN lines. For evaluation purposes, 184 consecutive cases of frozen sections (60), gastrointestinal pathology (64), and urinary cytology (60) have been diagnosed at a distance using the system, and the telediagnosis obtained in this way has been compared with the traditional microscopic diagnosis. Diagnostic agreement ranged from $90 \%$ in urinary cytology to $100 \%$ in frozen sections. The results obtained suggest that such a system can be considered a useful tool for supporting the pathology practice in isolated hospitals.

\section{Introduction}

Telepathology is a subspecialty of telemedicine aimed at supporting the pathologist's practice by means of telematic tools.

A number of different technical approaches has been developed, to solve different needs related to the practice of pathology at a distance, which is usually considered to include remote consultation $[1,18]$, intraoperative telediagnosis $[15,17,20]$, quality control $[8,10$,

\footnotetext{
*Correspondence to: Dr. Vincenzo Della Mea, Institute of Pathology, University of Udine, p.le S.Maria della Misericordia, 33100 Udine, Italy. Fax: +39 432 559420; E-mail: dellamea@dimi. uniud.it.
}

13], distant education [2,11], and remote image analysis $[7,12]$. Some of these tasks need real-time communications, some do not, leading to different solutions.

One of the most regarded applications, with foreseeable success, is the intraoperative telediagnosis to be applied between small hospitals without pathology service and pathology services located elsewhere, for giving support to an isolated pathologist sometimes needing consultation, or even to completely transfer the diagnostic work in another institute. This could lead either to better care or expense reductions.

Intraoperative telediagnosis needs real-time methods for communication, because it is an urgent task, to be carried out as fast as possible; thus it is not possible to adopt the so-called store-and-forward methods $[3,16]$, already proposed for remote consultation and quality control.

There are many technological ways to approach real-time telepathology, ranging from static to dynamic-robotic solutions [15]. Real-time static systems are based on the delivery of still images in near-realtime; in dynamic systems, real-time video from the microscope is sent to the remote observer. No matter whether the system is static or dynamic, the microscope can be operated either directly by a physician or technician, or remotely by the observer. In the former case, the selection of fields is made necessarily by the local operator, which can be guided vocally by the remote observer. In the latter case, a robotized microscope is used, together with a further software module for microscope operation; this way, a robotic - static or dynamic - telepathology system can be obtained. In particular, dynamic robotic telepathology allows the specimen's observation in real-time with the completely remote guide of the microscope, in a way similar to a normal session at the optical microscope. 
Dynamic telepathology is usually implemented by means of videoconferencing or videoconferencing-like protocols, from which image characteristics are inherited; this means that images have a resolution of up to $352 \times 288$ pixels, which is currently regarded as too low for diagnosis, whereas static telepathology may adopt higher resolutions. On the other side, dynamic systems allow for an easy location of fields during diagnosis. This occurs in a way similar to the behaviour of the microscope, whilst static systems can reach similar facilities by including a so-called slide-preview function, a sort of tiled low magnification reconstruction of the whole specimen from which higher magnifications can be visualised.

The present work describes the diagnostic performance and the usability of a robotic telepathology system that incorporates dynamic as well as static features, with the possibility of choosing the most adequate modality at any time. The dynamic component can be used for locating fields and solving most of the diagnostic problems, while the static part can be used when the video image quality is not enough. The system has been tested on frozen section as well as on histologic and cytologic specimens, in order to investigate the range of its capabilities. In particular, attention has been drawn on the use of still images during a dynamic examination, in order to evaluate the added value given by such capability to a dynamic-robotic system.

\section{Methods}

Remote diagnosis has been carried out between the Institute of Pathology of the University of Udine, Italy, and the $60 \mathrm{~km}$ far Laboratory of Pathology of the City Hospital of Tolmezzo, Italy, located on the mountain area in the north of Udine. The Hospital of Tolmezzo has a surgical service, but it does not have a permanent pathology service, which is rather provided by an external consultant pathologist, coming twice a week from the Institute of Pathology of Udine. This of course allows only for scheduled surgery.

\subsection{The cases}

184 histological and cytological cases were remotely analysed using the telepathology system between the Laboratory of Pathology of Tolmezzo and the Institute of Pathology of Udine, during three phases: 60 frozen sections, 64 gastrointestinal biopsies and 60 urinary smears, all unselected. Gastrointesti-
Table 1

A summary of organs involved in frozen section cases

\begin{tabular}{lc}
\hline Organ & Cases \\
\hline breast & 18 \\
ovary & 10 \\
lymph node & 10 \\
pharynx & 3 \\
small intestine & 3 \\
bone & 2 \\
brain & 2 \\
testis & 2 \\
oesophagus & 1 \\
kidney & 1 \\
bladder & 1 \\
liver & 1 \\
uterus & 1 \\
omentum & 2 \\
stomach & 1 \\
thymus & 1 \\
lung & 1 \\
Total & 60 \\
\hline
\end{tabular}

nal biopsies and urinary smears were consecutively obtained from archives, while frozen sections were all the daily workload at the Hospital of Tolmezzo during the time of experimentation, and partly consecutively obtained from the archives (Table 1). The cases from daily routine were previously diagnosed (including macroscopic handling and examination) by a different pathologist, in order not to give implications on surgical treatment. Macro-examination data were communicated to the remote pathologist when available in the archive records.

Gastrointestinal biopsies diagnosis was categorised as: no lesions, inflammatory findings, benign lesions and malignant lesions. The diagnoses of urinary smears were distinguished among insufficient material, not clinically significant (no lesions and inflammatory findings) and clinically significant (atypical issues and malignancy).

\subsection{The telepathology system}

The system used for telediagnosis was a preliminary version of the Migra telepathology workstation (Olympus, Germany), which features both static and dynamic subsystems, in connection with a Sony 3CCD videocamera (Fig. 1). The microscope was a Provis AX70 fully robotized microscope (Olympus, Japan), which has motor-driven stage, objectives, illumination, focus and autofocus. 


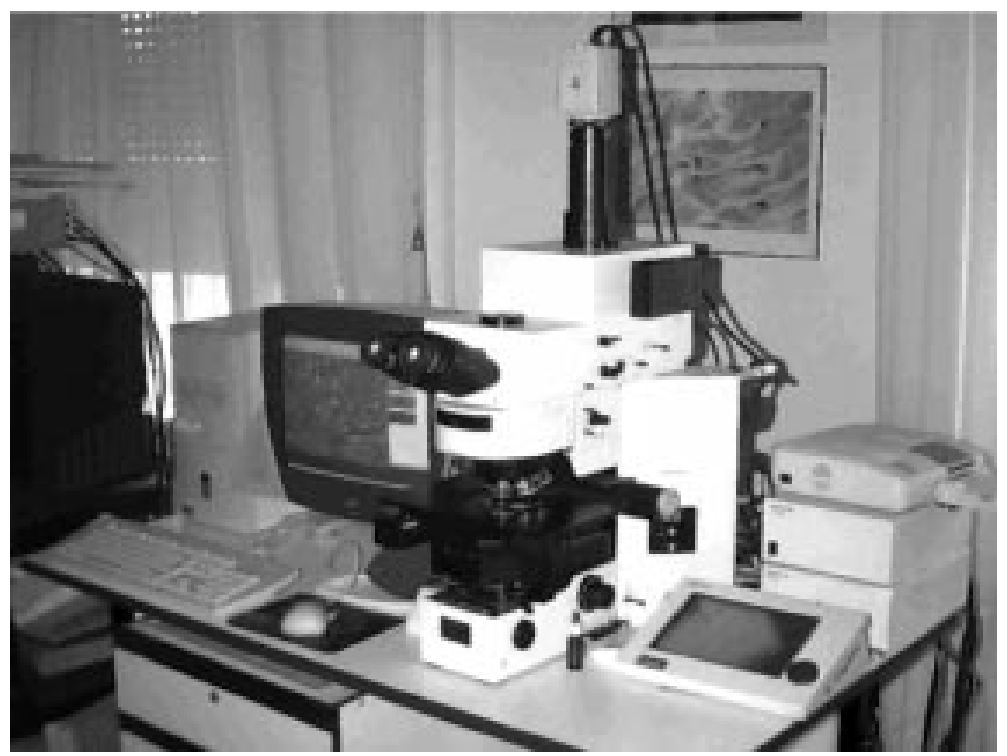

Fig. 1. The complete Migra telepathology system.

The Vision\&Live dynamic subsystem (IAT, Switzerland) is based on the H320 videoconference protocol, and uses up to 3 ISDN basic rate lines for transmission of real-time audio and video (equivalent to $384 \mathrm{Kbit} / \mathrm{s}$ in the Euro-ISDN standard). It allows for selection of video sources, which are usually two, one linked at the microscope camera, and the other at a desktop camera useful for videoconferencing between local and remote operator, together with an audio connection. The data channel of the system is used for remotely driving the robotic microscope, when available.

The static subsystem, developed by Bildanalysis Systems (Sweden), is based on NetMeeting protocols (Microsoft, USA) and implements a shared workspace with cursor sharing and drawing and measure tools. Additional modules for case storage, management and delivery through email are present. The static module uses another ISDN basic rate line $(128 \mathrm{Kbit} / \mathrm{s})$ for image transmission.

The remote guide of the microscope stage occurs by means of a joystick; all other functions can be accessed through software buttons.

Static and dynamic subsystems are fully integrated, and receive the video input from the same source, although through two framegrabbers with different quality and resolution. The remote pathologist usually observes the glass slide by means of the dynamic module, navigating around it using the joystick, and changing magnification and illumination when needed, thus seeing in the video monitor almost the same as if he/she were directly at the microscope. If the image quality is not satisfying, he or she can then request for a static image, which temporarily substitutes the video on the same monitor. The image can also be stored for further reference, saving also the coordinates of the acquisition.

\subsection{Statistical analysis}

For each diagnostic session, the telediagnosis has been recorded, together with the time for microscopic diagnosis and the number and magnification of static images requested during diagnosis. The diagnosis at the microscope for each case has also been recorded, including the final diagnosis for frozen sections.

In the first phase, Tolmezzo was the referring telepathology workstation, while Udine was the consultant workstation, where the pathologist formulated the diagnosis by visualising images on the monitor of the computer. In second and third phases the roles were inverted, so Udine was the referring station and Tolmezzo the consultant station.

When communication errors or software problems occurred, a description of the problem has been recorded; comments on usability have been recorded too. Such reports have then been used by the developers to improve the system.

Diagnostic agreement has been described as percentage of corresponding traditional and telepathologic diagnoses; the precision of estimate for diagnostic accuracy has been given by means of approximate 
Table 2

Sensitivity, specificity, positive predictive value and negative predictive value of the method

\begin{tabular}{lcccc}
\hline Cases & Sensitivity & Specificity & $\begin{array}{c}\text { Positive } \\
\text { predictive value }\end{array}$ & $\begin{array}{c}\text { Negative } \\
\text { predictive value }\end{array}$ \\
\hline $\begin{array}{l}\text { Frozen sections } \\
\text { Gastrointestinal }\end{array}$ & $100 \%$ & $100 \%$ & $100 \%$ & $100 \%$ \\
$\quad \begin{array}{c}\text { pathology } \\
\text { Urinary cytology }\end{array}$ & $100 \%$ & $100 \%$ & $100 \%$ & $97 \%$ \\
Total & $88 \%$ & $100 \%$ & $100 \%$ & $100 \%$ \\
\hline
\end{tabular}

$95 \%$ confidence intervals (CI), as suggested in [9]. We also calculated sensitivity, specificity, positive predictive value and negative predictive value of the method for all cases, and separately for the three categories. Descriptive statistics of time and static images needed for each case has been given in the form of median and quartiles. Pearson correlation and linear regression have been applied to evaluate the dependence of static images and time.

\section{Results}

\subsection{The diagnoses}

The evaluation of diagnostic accuracy in all cases showed an agreement of 94\% (CI: 92.2-96.8\%). Diagnostic agreement for frozen sections was $100 \%$ (CI: 97.5-100\%).

In gastrointestinal pathology, the agreement was 92.2\% (CI: 85.6-98.8\%), with 5 discordant cases: 2 false negatives and 3 minor failures.

The diagnoses of urinary smears had a total agreement of $90 \%$ (CI: 82.4-97.6\%), because 6 telepathological diagnoses of atypical smears were diagnosed as inflammatory findings or insufficient material at the microscope.

Table 2 shows sensitivity, specificity, positive predictive value and negative predictive value of the method for all cases, and separately for the three categories.

Tables 3, 4 and 5 describe respectively the case composition of the frozen section, gastrointestinal pathology and urinary cytology sets of cases.

\subsection{The time}

The overall median time for the telediagnosis was $4^{\prime} 00^{\prime \prime}\left(2^{\prime}-5^{\prime} 45^{\prime \prime}\right)$. In frozen sections and gastrointestinal biopsies, the median time needed was $3^{\prime} 00^{\prime \prime}$
Table 3

Case composition for the frozen sections set

\begin{tabular}{lc}
\hline Pathology & Cases \\
\hline Non-neoplastic diseases & 21 \\
Benign diseases & 11 \\
Malignant diseases & 28 \\
Total & 60 \\
\hline
\end{tabular}

Table 4

Case composition for the gastrointestinal pathology set

\begin{tabular}{lc}
\hline Pathology & Cases \\
\hline No lesions & 7 \\
Active chronic gastritis & 42 \\
Acute and chronic gastritis & 2 \\
Tubular adenoma & 6 \\
Adenocarcinoma & 5 \\
Lymphoma & 1 \\
Inadequate & 1 \\
Total & 64 \\
\hline
\end{tabular}

Table 5

Case composition for the urinary cytology set

\begin{tabular}{lc}
\hline Pathology & Cases \\
\hline Negative & 16 \\
Inflammatory findings & 24 \\
Cytological alterations NOS & 3 \\
Atypia & 10 \\
Transitional carcinoma & 5 \\
Inadequate & 2 \\
Total & 60 \\
\hline
\end{tabular}

$\left(2^{\prime} 00^{\prime \prime}-5^{\prime} 00^{\prime \prime}\right)$; finally, urinary cytology was diagnosed at distance in a median time of $4^{\prime} 30^{\prime \prime}\left(3^{\prime} 00^{\prime \prime}-8^{\prime} 00^{\prime \prime}\right)$.

The highest time was recorded in a cytological case, with 23 minutes, which involved the transfer of 4 static images.

In one occasion, software problems with the need to restart the computer led to 18 minutes for a frozen section diagnosis. 


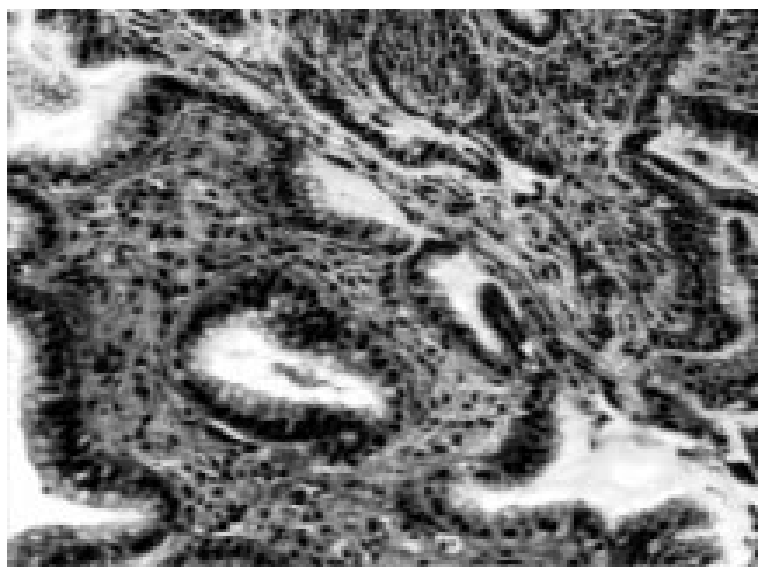

(A)

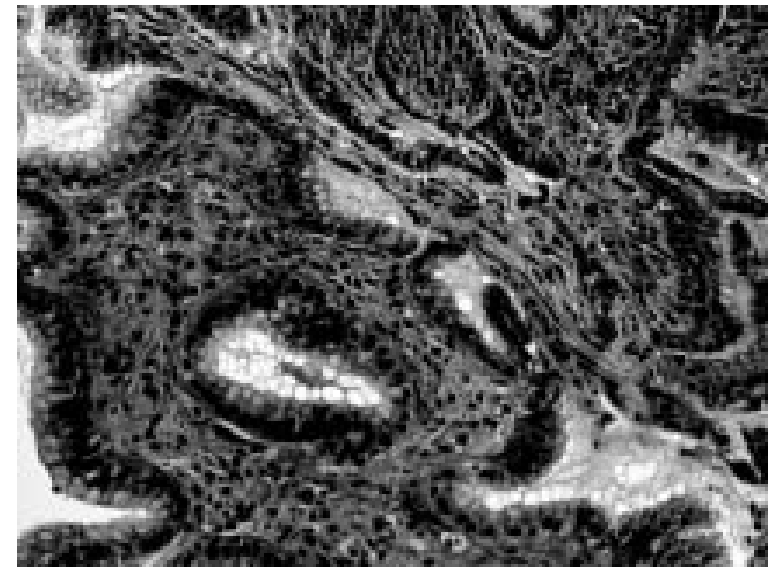

(B)

Fig. 2. The same field (gastrointestinal biopsy, HE, 20×) acquired through the dynamic (A) and through the static (B) subsystem.

\subsection{The static images}

An overall median number of $1(0-2)$ static images was transferred for each case. In particular, frozen sections needed a median number of 1 (1-2.75) images, gastrointestinal biopsies $1(0.25-2)$ and urinary cytology $1(0-1)$. Overall, 58 cases $(31 \%)$ have been diagnosed without the use of still images, while 71 cases (39\%) needed only one image.

The overall time needed for transmission is slightly correlated to the number of static images transmitted $(r=0.54)$. Applying the linear regression analysis, this results in the following parameters:

$$
\text { total time }=2^{\prime} 53^{\prime \prime}+1^{\prime} 22^{\prime \prime} * \text { static images }
$$

$$
\left(r^{2}=0.29\right) \text {. }
$$

This could be interpreted as a part of the time being devoted to glass slide navigation by means of the dynamic subsystem, with a further part devoted to static image transfer and analysis. This in turn fits in the diagnostic behaviour patterns studied by Tsuchihashi et al. [23].

The relationship between failed cases and number of static images has also been investigated, discovering a slight and not significant increase in the average number of images in such cases. This can be due perhaps to some difficulties in the interpretation, in turn explainable to either the case difficulty or the glass slide quality.

Figure 2 shows the same field acquired through the dynamic (Fig. 2A) and static (Fig. 2B) subsystems; as can be seen, the image quality is substantially different.
Comments were transferred to developers, in order to enhance the usability of the system. As a result, a new version has been released, which also includes the slide preview and an alternative interaction system, based on the mouse. Occasional problems have been registered with ISDN lines, in particular when establishing the connection; this kind of problems should be taken into account particularly when dealing with frozen sections, because of the urgency needed for their diagnosis.

\section{Discussion}

The experimentation carried out in this study allowed to evaluate the diagnostic accuracy of a dynamicrobotic telepathology workstation, as already made by others with similar results $[6,16,21]$. The study involved three pathology fields; in the most important of these for its applicative possibilities, i.e., frozen sections, the system showed an extremely good behaviour, from the point of view of diagnostic agreement as well as of the time needed for diagnosis.

The other two pathology areas, included in the study for completeness of evaluation, gave some additional information.

In gastrointestinal pathology the agreement results are slightly better than those previously obtained using static telepathology [4]. In particular, two adenocarcinomas were diagnosed as chronic gastritis during the telepathologic session. In one case, the carcinoma was focal with wider areas of severe dysplasia and microinvasion. In the second case the carcinoma was present 
only in one of the fragments on the slide, while the others shown only the presence of chronic gastritis. Most probably the discrepancy can be explained as follows: it is possible that, during the telepathologic session, the pathologist didn't check the whole slide, and missed the cancer.

A solution for this has been proposed as a support to the diagnostic activity in the telepathology software: a graphical mark of already visited areas on the specimen preview, which helps the pathologist in effectively examining the whole sample. Such a technical feature, present in other systems [5], is of help also in telecytological examinations, and has been implemented in the last software release.

In cytology, the time needed for the diagnosis is higher than that needed in the other two fields, because the evaluation of single cell morphology is the most crucial diagnostic step, and often material is spread around the glass slide. This effect has already been reported by others [22]. However, diagnostic discordance was mainly due to minor errors.

The studied system is particular, as it provides for both dynamic and static telepathology, the latter thought as a way for overriding the classical resolution problems of videoconferencing-based dynamic systems. In fact, just a moderated number of still images are exchanged in many cases, giving a small time overhead but also an important possibility in respect to purely dynamic systems. The median time needed for a diagnosis is similar to the lowest times reported in the literature for realtime systems [20,21], and lower than the results of static-robotic systems $[5,17]$, due to the locator functionality given by the real-time video capabilities. This, in our opinion, pushes towards integration between both modalities, in order to obtain both speed and, when necessary, the higher precision given by static images.

During the experimentation, some problems have been experienced with the communication lines, which are not so reliable as expected.

The adoption of telepathology should be preceded by an evaluation of savings that can be eventually reached in respect to more traditional solutions. However, when the aim is to give more support to an isolated pathologist, there are surely benefits on the diagnostic quality, which are difficult to be directly evaluated by means of economical analysis.

Besides of this, the main problem that makes the adoption of telepathology as a mean for doing a completely remote diagnostic service difficult, is the macroscopic sampling of the surgical specimen. In fact, although there are experiences where the surgeon is responsible of such a task [16], pathologists seem not to agree to let others do this important part of their work [13]. In addition, legal aspects coming from the shared responsibility on the diagnosis should be studied.

The overall results allow us to consider the robotized telemicroscopy system as a helpful tool for delivering pathology services to isolated hospitals, and thus to give an health care service of the same quality as that given by central hospitals to otherwise underserved population.

\section{Acknowledgements}

This work was partially supported by a grant of the Consorzio Universitario del Friuli. We thank Olympus Italy for the support, the personnel of the Hospital of Tolmezzo for the kind collaboration during the experimentation and Dr Sabrina Guerra for her significant help in reviewing the article.

\section{References}

[1] C.A. Beltrami and V. Della Mea, Second opinion consultations through the Internet. A three years experience, Advances in Clinical Pathology 2 (1998), 146-148.

[2] E.M. Brebner, J.A. Brebner, J.N. Norman, P.A. Brown, H. Ruddick-Bracken and J.H. Lanphear, Intercontinental postmortem studies using interactive television, Journal of Telemedicine and Telecare 3 (1997), 48-52.

[3] V. Della Mea, Store-and-forward telepathology, in: European Telemedicine 1998/99, B. Hernandez and R. Wootton, eds, EHTO/RSM Press/Kensington Publications, London, 1999, pp. 74-76.

[4] V. Della Mea, S. Forti, F. Puglisi, P. Bellutta, N. Finato, P. Dalla Palma, F. Mauri and C.A. Beltrami, Telepathology using Internet multimedia electronic mail: remote consultation on gastrointestinal pathology, Journal of Telemedicine and Telecare 2 (1996), 28-34.

[5] F. De Michelis, C. Eccher, C. Clemente, G. Migliore, P. Dalla Palma and S. Forti, A feasibility study of a static-robotic telepathology system for remote diagnosis, Advances in Clinical Pathology 2 (1998), 138-139.

[6] B.E. Dunn, U.A. Almagro, H. Choi, N.K. Sheth, J.S. Arnold, D.L. Recla, E.A. Krupinski, A.R. Graham and R.S. Weinstein, Dynamic-robotic telepathology: Department of Veterans Affairs feasibility study, Human Pathology 28 (1997), 8-12.

[7] S. Forti, C. Eccher, R. Visentin, V. Della Mea, F. Puglisi, M. Barbareschi, P. Bellutta, C.A. Beltrami and P. Dalla Palma, Distributed laboratory for remote image analysis in immunohistochemistry, Journal of Telemedicine and Telecare 3(S1) (1997), 94A. 
[8] G. Haroske, W. Meyer, D. Kunze and A. Böcking, Quality control measures for DNA image cytometry in a telepathology network, Advances in Clinical Pathology 2 (1998), 143-145.

[9] R. Harper and B. Reeves, Reporting of precision of estimates for diagnostic accuracy: a review, BMJ 318 (1999), 1322-1323.

[10] K. Kayser and G. Kayser, Basic aspects of and recent developments in telepathology in Europe, with specific emphasis on quality assurance, Analytical and Quantitative Cytology and Histology 21 (1999), 319-328.

[11] J. Klossa, J.C. Cordier, G. Flandrin, C. Got and J. Hemet, A European de facto standard for image folders applied to telepathology and teaching, International Journal of Medical Informatics 48 (1998), 207-216.

[12] K.D. Kunze, A. Böcking, G. Haroske, K. Kayser, W. Meyer and M. Oberholzer, Remote quantitation in the framework of telepathology, Advances in Clinical Pathology 2 (1998), 141143.

[13] F.J.W.M. Leong, A.K. Graham, P. Schwarzmann and J.O'D. McGee, Controlled clinical trials of robotic interactive telepathology in the national external quality assurance scheme, Journal of Pathology 187(Suppl. 1) (1999), 5A.

[14] T. Mairinger, T.T. Netzer, W. Schoner and A. Gschwendter, Pathologists' attitudes to implementing telepathology, Journal of Telemedicine and Telecare 4 (1998), 41-46.

[15] I. Nordrum, Real-time diagnoses in telepathology, Advances in Clinical Pathology 2 (1998), 127-131.

[16] I. Nordrum and T.J. Eide, Remote frozen section service in Norway, Archives d'Anatomie et de Cytologie Pathologiques 43 (1995), 253-256.
[17] M. Oberholzer, H.R. Fischer, H. Christen, S. Gerber, M. Bruhlmann, M.J. Mihatsch, T. Gahm, M. Famos, C. Winkler, P. Fehr, H.J. Hosch and L. Bächtold, Telepathology: frozen section diagnosis at a distance, Virchows Archiv $\mathbf{4 2 6}$ (1995), 3-9.

[18] D.A. Perednia, Reinventing telemedicine: store-and-forward applications, Telemedicine and Telehealth Networks 2 (1996), $15-18$.

[19] S. Raab, R.A. Robinson, T.E. Snider, H.L. McDaniel, J.D. Sigman, C.J. Leigh and P.Q. Thomas, Telepathologic review: utility, diagnostic accuracy, and interobserver variability on a difficult case consultation service, Modern Pathology 10 (1997), 630-635.

[20] Y. Shimosato, Y. Yagi, K. Yamagishi, K. Mukai, S. Hirohashi, T. Matsumoto and T. Kodama, Experience and present status of telepathology in the National Cancer Center Hospital, Tokyo, Zentralblatt für Pathologie 138 (1992), 413-417.

[21] P. Schwarzmann, B. Binder, R. Klose and M. Kaeser, Histkom - evaluation of active telepathology in fieldtests, Advances in Clinical Pathology 2 (1998), 135-138.

[22] P. Schwarzmann, U. Schenck, B. Binder and J. Schmid, Is todays telepathology equipment also appropriate for telecytology? A pilot study with pap and blood smears, Advances in Clinical Pathology 2 (1998), 176-178.

[23] Y. Tsuchihashi, T. Mazaki, K. Nakasato, M. Morishima, H. Nagata, I. Tofukuji, H. Shirakata, K. Naito and Y. Akasaka, The basic diagnostic approaches used in robotic still-image telepathology, Journal of Telemedicine and Telecare 5(S1) (1999), 115-117. 


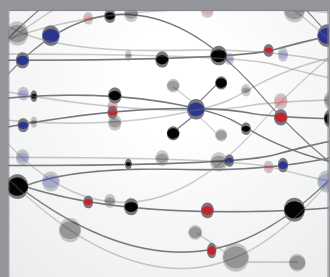

The Scientific World Journal
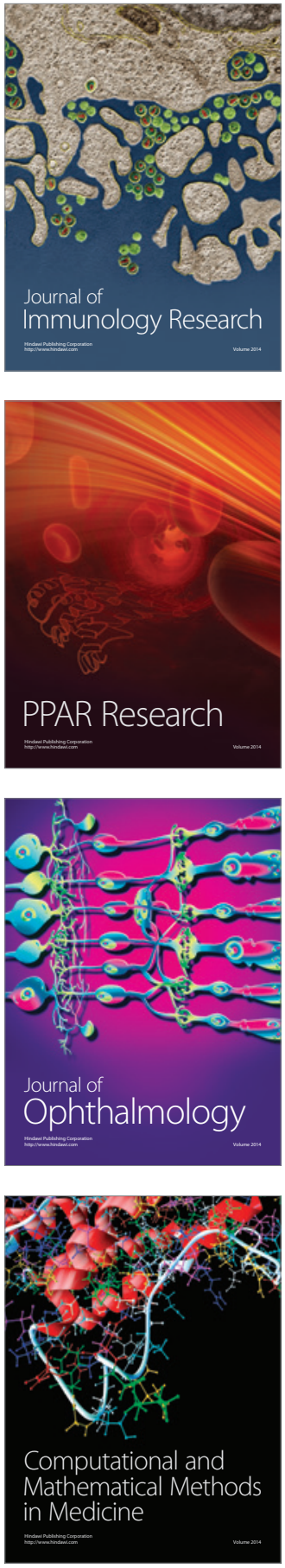

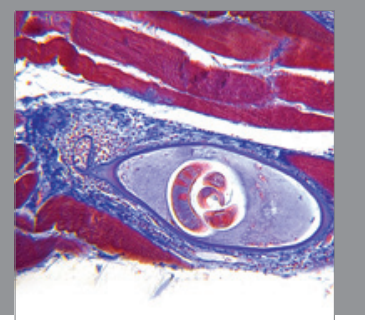

Gastroenterology

Research and Practice
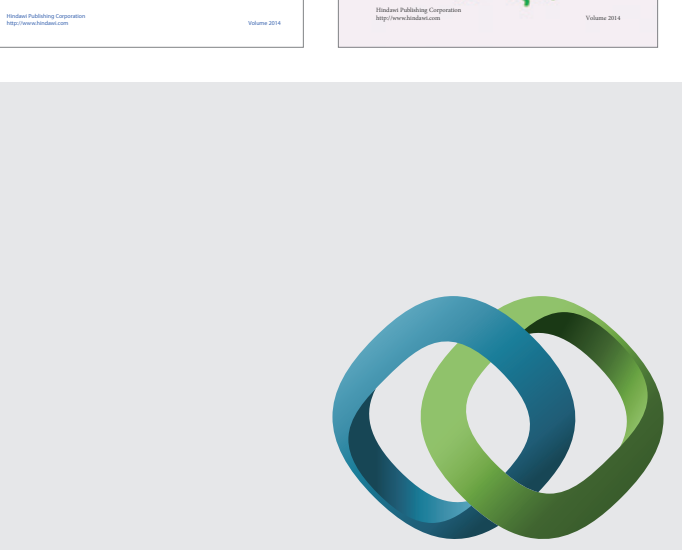

\section{Hindawi}

Submit your manuscripts at

http://www.hindawi.com
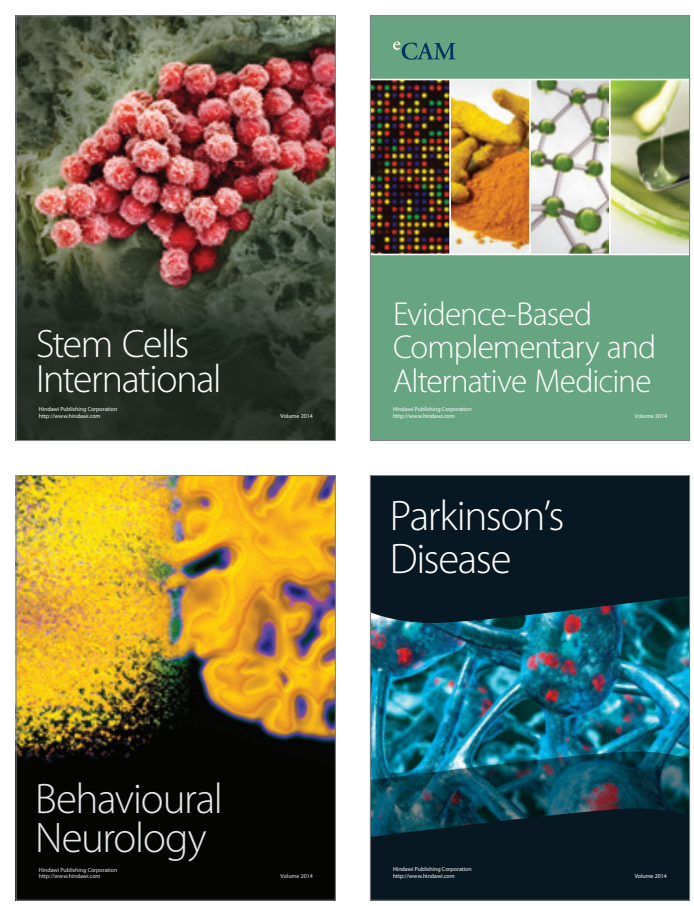

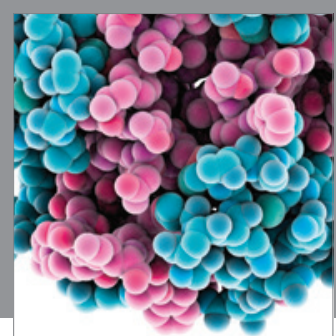

Journal of
Diabetes Research

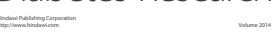

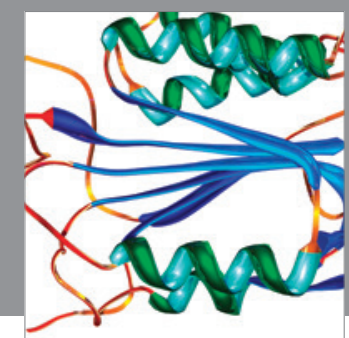

Disease Markers
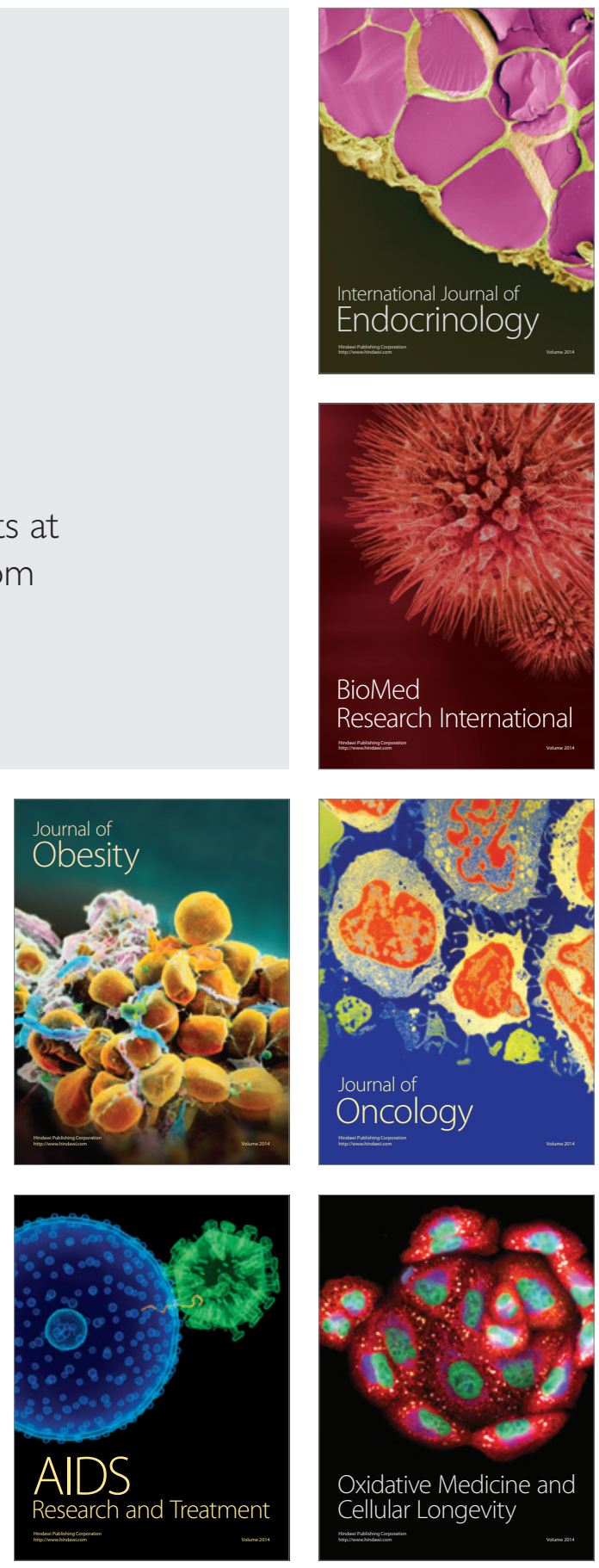